\title{
Distribution of Prostate Cancer for Optimized Biopsy Protocols ${ }^{1 *}$
}

\author{
Jianchao Zeng ${ }^{\mathrm{a}}$, John J. Bauer ${ }^{\mathrm{b}}$, Ariela Sofer ${ }^{\mathrm{c}}$, Xiaohu Yao ${ }^{\mathrm{a}}$, \\ Brett Opell ${ }^{\mathrm{d}}$, Wei Zhang ${ }^{\mathrm{e}}$, Isabell A. Sesterhenn ${ }^{\mathrm{e}}$, Judd W. Moul ${ }^{\mathrm{b}, \mathrm{f}}$, \\ John Lynch ${ }^{\mathrm{d}}$, and Seong K. Mun ${ }^{\mathrm{a}}$ \\ ${ }^{a}$ Imaging Science and Information Systems Center (ISIS), \\ Department of Radiology, Georgetown University Medical Center, \\ Washington,DC 20007 \\ zeng@isis.imac.georgetown.edu (J. Zeng) \\ http://www.simulation.georgetown.edu \\ ${ }^{\mathrm{b}}$ Urology Service, Department of Surgery, \\ Walter Reed Army Medical Center, \\ Washington, DC 20307-5001 \\ ${ }^{c}$ Department of Operations Research and Engineering, \\ School of Information Technology and Engineering, \\ George Mason University, \\ Fairfax, Virginia 22030 \\ ${ }^{\mathrm{d}}$ Urology Division, Department of Surgery, \\ Georgetown University Medical Center, \\ Washington, DC 20007 \\ ${ }^{\mathrm{e}}$ Department of Genitourinary Pathology, \\ Armed Forces Institute of Pathology (AFIP), \\ Washington, DC 20306-6000 \\ ${ }^{\mathrm{f}}$ Center for Prostate Disease Research (CPDR), Department of Surgery, \\ Uniformed Services University of the Health Sciences (USUHS), \\ Bethesda, Maryland 20814-4799
}

\begin{abstract}
Prostate cancer is the leading cause of death for American men. The gold standard for diagnosis of prostate cancer is transrectal ultrasound-guided needle core biopsy. Unfortunately, no imaging modality, including ultrasound, can effectively differentiate prostate cancer from normal tissues. As a result, most current prostate needle biopsy procedures have to be performed under empiric protocols, leading to unsatisfactory detection rate. The goal of this research is to establish an accurate 3D distribution map of prostate cancer and develop optimized biopsy protocols. First, we used real prostate specimens with localized cancer to reconstruct $3 \mathrm{D}$ prostate models. We then divided each model into zones based on clinical conventions, and calculate cancer presence in each zone. As a result, an accurate 3D prostate cancer distribution map was developed using 281 prostate models. Finally, the linear programming approach was used to optimize biopsy protocols using anatomy and symmetry constraints, and the optimized protocols were developed with various criteria.
\end{abstract}

* This research has been supported, in part, by The Whitaker Foundation, The US Army Medical Research and Materiel Command, and The Center for Prostate Disease Research. S.L. Delp, A.M. DiGioia, and B. Jaramaz (Eds.): MICCAI 2000, LNCS 1935, pp. 287-296, 2000. (c) Springer-Verlag Berlin Heidelberg 2000 


\section{Introduction}

In the United States, approximately 200,000 new cases of prostate cancer are detected annually, and about 40,000 men die of prostate cancer each year. Transrectal Ultrasonography (TRUS) guided systematic needle biopsy of the prostate has been widely used clinically for the diagnosis and staging of prostate carcinoma. Due to the low resolution of the ultrasound images, however, a urologist can hardly differentiate abnormal tissues from normal ones during the biopsy. Therefore a number of protocols have been developed to help urologists perform prostate needle biopsy. A biopsy protocol designates locations of the needles on the prostate as well as the number of needles to use. The most common protocol currently used clinically is the systematic sextant biopsy protocol ${ }^{8}$.

Recent studies, however, have shown that the accuracy of currently used biopsy techniques needs to be improved ${ }^{1,4}$. These studies show that a significant number of patients who have prostate cancer are not diagnosed at their initial biopsy. As a result, there are a significant number of prostate cancers that are detected on repeat biopsies. If biopsy protocols can be optimized to increase the chances of detecting prostate cancer, the value of these new protocols as a diagnostic tool will be significant.

A number of researchers have investigated techniques for improving the accuracy of biopsy protocols ${ }^{5,15}$. Eskew et al. introduced a 5-region biopsy protocol in which additional lateral and midline biopsies were added systematically to the traditional sextant biopsy ${ }^{6}$. Our group found that the 5-region protocol showed a statistically significant advantage over the sextant method based on simulation results of 89 patients with cancer ${ }^{10}$. Chang et al. showed that lateral biopsies increase the sensitivity of prostate cancer detection ${ }^{3}$. We have also showed biopsy protocols that use laterally placed biopsies based upon a five region anatomical model are superior to the routinely used sextant prostate biopsy pattern ${ }^{2}$. Goto et al. suggested that new biopsy strategies could be developed based on probability maps of cancer distribution within the prostate ${ }^{7}$. But issues such as how these maps should be built and how new biopsy protocols could be derived from the maps remain to be investigated.

In this research, our goal is to establish an accurate 3D distribution map of prostate cancer, and to develop optimized biopsy protocols. In this paper, we first use real prostate specimens with localized cancer to reconstruct $3 \mathrm{D}$ prostate models. We then divide each model into zones based on clinical conventions, and calculate cancer presence in each zone. As a result, an accurate $3 \mathrm{D}$ prostate cancer distribution map is developed using 281 prostate models. Further, the linear programming approach is used to optimize biopsy protocols using anatomy and symmetry constraints, and optimized protocols are developed with various clinical criteria.

\section{Reconstruction of 3D Prostate Models and 3D Distribution Map of Prostate Cancer}

We have developed a deformable modeling technique for the reconstruction of 3D prostate surface models based on step-sectioned whole-mounted radical 
prostatectomy specimens with localized cancers ${ }^{14,15,11}$. Currently, two hundred and eighty-one 3D prostate models have been so far reconstructed.

In order to establish optimized prostate needle biopsy protocols, we have investigated the spatial distribution of the prostate cancers with the $3 \mathrm{D}$ models in such a way that will help directly determine an optimized protocol for improved cancer detection. Based on the low-resolution nature of currently available ultrasound imaging and the current clinical conventions for TRUS guided prostate biopsy, we have divided a prostate gland into different zones (instead of directly dealing with individual voxels) that are accessible by the urologists under the guidance of ultrasound images. By checking cancer presence inside each of these zones using a large number of 3D prostate models, we can develop an overall spatial distribution map of prostate cancer among the various zones. And based on this distribution map, an optimal biopsy protocol can be developed.

This approach is characterized by its precision in determining prostate cancer distribution throughout the whole prostate gland. Since there is no artificial deformation made to the individual prostate models during the process, the resulting information on prostate cancer location and distribution should precisely reflect the original cancer presence. Unlike other deformation-based approaches, our developed approach does not make any approximation and therefore no error is introduced in the results. In addition, the different sizes of individual prostate models will not affect the results of this approach since it uses the same and consistent concept of zones as that used by urologists. That is, although a larger prostate will certainly have a larger size for each of its zones, each corresponding zone in different prostate models carries the same spatial meaning and is treated equally regardless of its size.

\section{(1) Algorithm for Dividing Zones and Detecting Cancers in the Zones}

The algorithm is composed of 2 steps: a 3D-based checking and a 2D-based checking. The 3D-based checking module draws attention to each zone that can precisely identify the presence or non-presence of a cancer inside its scope. The 2D-based checking is used to identify if each zone intersects with cancers or is totally contained inside a large cancer by reviewing 2D section layers of both the cancers and the zone. The 3D-based checking is a quick process dealing with simple situations while the 2D-based checking is a more intensive search process for any complex cases that are left over by the 3D-based checking step.

\section{- 3D-Based Checking}

The 3D-based checking is performed to quickly make decisions on the presence of a cancer inside a zone using the 3D bounding box information. Only two distinct cases are considered here: a cancer is totally inside a zone, or a cancer is totally outside of a zone. When a cancer completely resides in a zone, every vertex of the cancer bounding box is inside the zone space. In implementation, we have used an 8-bit value to test the inside property of each cancer, while every bit corresponds to every vertex (1: inside, 0 : outside). Initially the value is set to $0 x 00000000$ and the test is performed for each zone. If the test ends up with a value of $0 \times 11111111$, the zone can be marked as positive. Otherwise, further investigation is needed to make a decision. When a cancer is completely outside the zone, we assume that the space is partitioned 
into two sides by each zone wall. All vertices of the cancer bounding box should reside in the same side if the cancer is totally outside of the zone. We need to perform six tests, one for each zone wall. In implementation, we have used a 6-bit value to test the same-side property. Each zone is set to 0x000000 initially and every vertex of the cancer bounding box updates this value after the test. The final result of this test is obtained by an "AND" operation of the eight values. If a zone ends up with a value not equal to $0 \mathrm{x} 000000$, the cancer is recognized as existing outside of this zone scope, and the zone is thus marked as negative. In the case of multiple cancers, this process is performed with respect to each of them, and all the results will be combined by an "AND" operation. Any result other than 0x000000 will lead to a negative zone.

\section{- 2D-Based Checking}

The 2D-based checking module deals with those zones that cannot be quickly determined in the 3D-based checking step. For each zone $C_{i}$ that needs 2D-based checking, first we need to identify which layers of the cancer can possibly intersect with $\mathrm{C}_{\mathrm{i}}$. That is, we need to find those layers of the cancer with a $\mathrm{Z}$ coordinate between $C_{\mathrm{i}}$.Maximum_Z_Value and $C_{\mathrm{i}}$.Minimum_Z_Value. If we can prove that at least one cancer intersects with the zone in any of these layers, this zone will then be marked as positive for containing cancer. Otherwise, if all applicable layers fail to yield such a proof, this zone will be marked as negative. A scan line algorithm is developed to find such a proof fast for each 2D layer.

The scan line algorithm starts from the left margin of the zone section, and keeps moving to the right end at a given step. The intersection between the scan line and the polygon edges of the cancer layer can be found quickly since the edges have already been sorted beforehand. If this intersection lies inside the zone section, the 2D-based checking is completed with positive result. Otherwise, the algorithm partitions the layer into two portions: one above and one below the zone. If the scan line has odd number of intersections with the cancer edge in each portion, the cancer polygon edge must contain, at least in part, the zone section, and thus the zone will be marked as positive. If, on the other hand, the intersections are in even number in each portion, the cancer might be in a concave shape here without containing the zone, and the algorithm will continue the scanning process until the end.

\section{(2) A Comprehensive 48 Division Scheme}

Obviously, the more divisions the more detailed information we can get for cancer distribution. At the same time, however, in order for the distribution information useful directly to the urologists, a division scheme should allow its zones to be recognizable and accessible by them under the guidance of transrectal ultrasound probe used for prostate biopsy. Under such constraint, we have conducted a comprehensive 48 division of prostates, which is probably the maximum number of division we can get for the purpose of prostate biopsy.

In the 48-zone division, looking at a prostate in axial view, a prostate gland is divided into 4 symmetric compartments sideways, 4 symmetric compartments vertically, and 3 compartments in depth. An example of a prostate model with 48 -zone division is shown in Figure 1. The size of the zones will vary with the size of a prostate model. A larger prostate ends up having a larger size for each of its 48 zones. This variation of 
zone size is the natural reflection of the original prostate, and it does not affect the accuracy of cancer distribution. The labeling of each zone follows the current clinical conventions. The four layers of zones parallel to the YZ plane (sagittal) are labeled along the positive $\mathrm{X}$ direction as: left lateral (11), left mid (lm), right mid (rm) and right lateral (rl), respectively. The three layers of zones parallel to XY plane (axial) are labeled along the positive $\mathrm{Z}$ direction as: base, mid and apex, respectively. Similarly, the four layers parallel to the XZ plane (coronal) are labeled along the positive $\mathrm{Y}$ direction as: posterior 1 (p1), posterior 2 (p2), anterior 1 (a1) and anterior 2 (a2), respectively. When a specific zone is labeled, the labels of the corresponding three layers are combined in the $\mathrm{X}-\mathrm{Y}-\mathrm{Z}$ order. For example, the zone ' $\mathrm{A}$ ' in Figure 1 is labeled as llp1-apex.

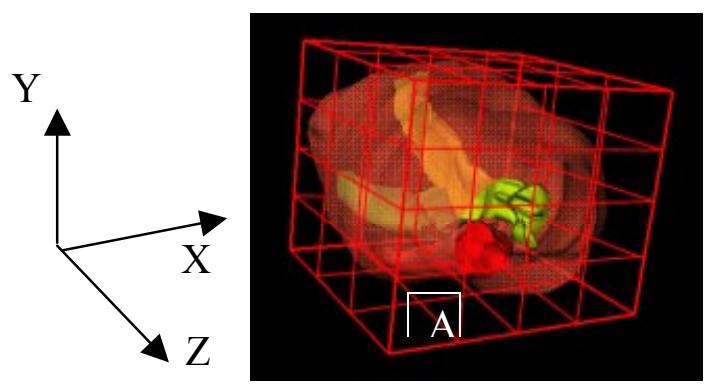

Figure 1. A prostate model with 48-zone division

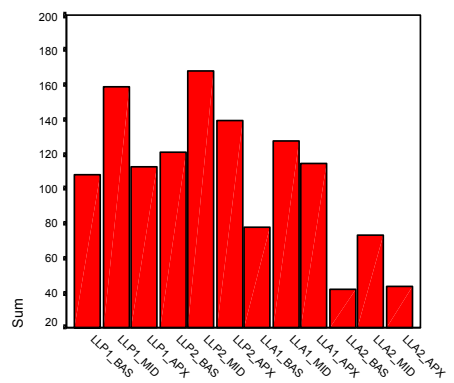

(a) Cancer distribution in left lateral layer

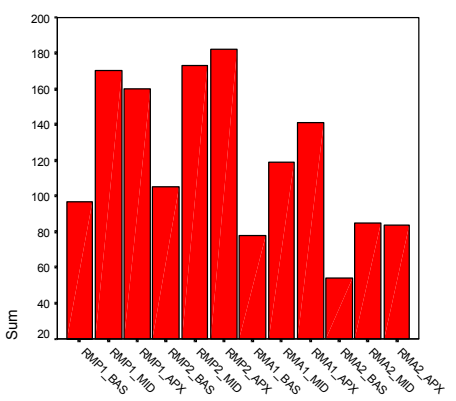

(c) Cancer distribution in right mid layer

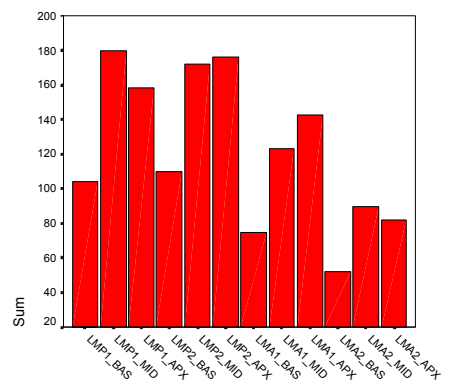

(b) Cancer distribution in left mid layer

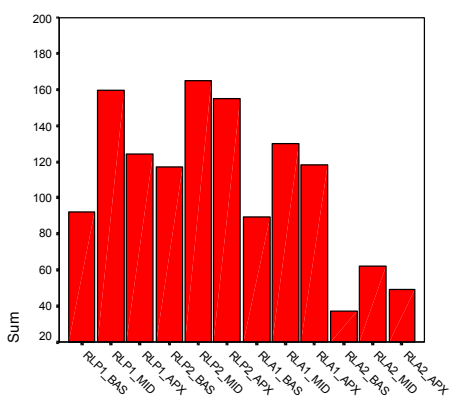

(d) Cancer distribution in right lateral layer

Figure 2. Cancer distribution presented in sagittal layers 
In order to investigate the cancer distribution inside the prostate gland, we have calculated the appearance of cancers in each zone of each of the 281 individual prostate models using the above algorithm. The results are presented in sagittal layers (Figure 2) and axial layers (Figure 3). From the statistical analysis, we have the following preliminary results: (1) The base zones (36.8\%) have a statistically significantly lower cancer distribution than mid zones $(56.3 \%)(p=0.001)$ and apical zones $(53.5 \%)(\mathrm{p}=0.001)$; (2) There is also a significantly higher cancer distribution in the posterior zones $(57.2 \%)$ than in the anterior zones $(40.5 \%)(p=0.001)$; (3) The mid zones $(56.3 \%)$ have a slightly significantly higher cancer distribution than the apical zones $(53.5 \%)(\mathrm{p}=0.032)$; and (4) There was no significant difference between the left zones $(49.2 \%)$ and right zones $(48.5 \%)(p=0.494)$. In order to develop an optimal biopsy protocol using the cancer distribution information, we have used the linear programming approach as described in the next section.

\begin{tabular}{|c|c|c|c|}
\hline $1 \mathrm{la} 2$ & $\operatorname{lma} 2$ & $\mathrm{rma} 2$ & $\mathrm{rla} 2$ \\
$14.95 \%$ & $18.51 \%$ & $19.22 \%$ & $13.17 \%$ \\
& & & \\
\hline $1 \mathrm{la} 1$ & $\operatorname{lma} 1$ & $\mathrm{rma} 1$ & $\mathrm{rla} 1$ \\
$27.76 \%$ & $26.6 \%$ & $27.76 \%$ & $31.67 \%$ \\
& & & \\
\hline $11 \mathrm{p} 2$ & $\operatorname{lmp} 2$ & $\mathrm{rmp} 2$ & $\mathrm{rlp} 2$ \\
$43.06 \%$ & $39.15 \%$ & $37.37 \%$ & $41.64 \%$ \\
\hline $11 \mathrm{p} 1$ & $\operatorname{lmp} 1$ & $\mathrm{rmp} 1$ & $\mathrm{rlp} 1$ \\
$38.43 \%$ & $37.01 \%$ & $34.52 \%$ & $32.74 \%$ \\
\hline
\end{tabular}

(a) Distribution in the base layer

\begin{tabular}{|c|c|c|c|}
\hline 1la2 & lma2 & rma2 & rla2 \\
$25.98 \%$ & $32.03 \%$ & $30.25 \%$ & $22.06 \%$ \\
& & & \\
\hline lla1 & lma1 & rma1 & rla1 \\
$45.20 \%$ & $43.77 \%$ & $42.35 \%$ & $46.26 \%$ \\
& & & \\
\hline $11 \mathrm{p} 2$ & $\operatorname{lmp} 2$ & $\mathrm{rmp} 2$ & $\mathrm{rlp} 2$ \\
$59.79 \%$ & $61.21 \%$ & $61.57 \%$ & $58.72 \%$ \\
\hline $11 \mathrm{p} 1$ & $1 \mathrm{mp} 1$ & $\mathrm{rmp} 1$ & $\mathrm{rlp} 1$ \\
$56.58 \%$ & $64.06 \%$ & $60.50 \%$ & $56.94 \%$ \\
\hline
\end{tabular}

(b) Distribution in the mid layer

\begin{tabular}{|l|c|c|c|}
\hline lla2 & lma2 & rma2 & rla2 \\
$15.66 \%$ & $29.18 \%$ & $29.89 \%$ & $17.44 \%$ \\
& & & \\
\hline lla1 & $\operatorname{lma} 1$ & $\mathrm{rma} 1$ & $\mathrm{rla} 1$ \\
$40.93 \%$ & $50.89 \%$ & $50.18 \%$ & $41.99 \%$ \\
\hline 1lp2 & lmp2 & $\mathrm{rmp} 2$ & $\mathrm{rlp} 2$ \\
$49.47 \%$ & $62.63 \%$ & $64.77 \%$ & $55.16 \%$ \\
& & & \\
\hline $11 \mathrm{p} 1$ & $\operatorname{lmp} 1$ & $\mathrm{rmp} 1$ & $\mathrm{rlp} 1$ \\
$40.21 \%$ & $56.23 \%$ & $56.94 \%$ & $44.13 \%$ \\
& & & \\
\hline
\end{tabular}

(c) Distribution in the apex layer

Figure 3. Cancer distribution presented in axial layers 


\section{Optimization of Biopsy Protocols}

The optimal biopsy protocols can be explored by solving a mathematical optimization problem. Here, we define an $m$ by $n$ matrix A such that,

$$
a_{i j}=\left\{\begin{array}{l}
1, \text { if prostate } \mathrm{i} \text { is found to have cancer in zone } \mathrm{j} \\
0, \text { otherwise }
\end{array}\right.
$$

where $m=281$ is the number of prostate models in the study, and $n=48$ is the number of zones in the $3 \mathrm{D}$ distribution map. We further define variables $x_{j}(\mathrm{j}=1, . ., \mathrm{n})$ by

$$
x_{j}=\left\{\begin{array}{l}
1, \text { if a biopsy is taken in zone } \mathrm{j} \\
0, \text { otherwise }
\end{array}\right.
$$

Then the minimum number of zones to be tested that would ensure, in theory, $100 \%$ cancer detection for the data set can be found by solving the following problem:

$$
\begin{array}{ll}
\text { Minimize } & \sum_{\mathrm{j}=1}^{\mathrm{n}} x_{j} \\
\text { subject to } & \sum_{\mathrm{j}=1}^{\mathrm{n}} a_{i j} x_{j} \geq 1, \quad \mathrm{i}=1, \ldots, \mathrm{m} \\
& x_{j}=0 \text { or } 1, \quad \mathrm{j}=1, \ldots, \mathrm{n}
\end{array}
$$

The problem of minimizing or maximizing a linear function subject to linear constraints is known as a linear program. The special case where the variables are restricted to take on integer values (as we have above) is called an integer program. Linear programs have been studied extensively ${ }^{12}$, and sophisticated software packages can often solve problems involving millions of variables and thousand constraints. Although integer programs are usually much harder to solve, recent advances have made it possible to solve many classes of problems effectively ${ }^{13}$. We can also define optimal biopsy protocols as those that provide maximum rate of cancer detection for a given number of biopsies. Again we define variables $y_{i}(\mathrm{i}=1, \ldots, \mathrm{m})$ by

$$
y_{i}=\begin{aligned}
& 1, \text { if cancer is detected for prostate } \mathrm{i} \\
& 0, \text { otherwise }
\end{aligned}
$$

Then the maximum number of prostates in the data set whose cancer would be detected by a $k$-biopsy protocol can be found by solving the following integer program:

$$
\begin{array}{ll}
\text { Maximize } & \sum_{\mathrm{i}=1} y_{i} \\
\text { subject to } & \sum^{\mathrm{n}} a_{i j} x_{j} \geq y_{i}, \quad \mathrm{i}=1, \ldots, \mathrm{m} \\
& \mathrm{j}=1 \\
& \mathrm{n}
\end{array}
$$




$$
\begin{aligned}
& \sum x_{j}=k \\
& \mathrm{j}=1 \\
& x_{j,} y_{i}=0 \text { or } 1, \mathrm{j}=1, \ldots, \mathrm{n}, \mathrm{i}=1, . ., \mathrm{m}
\end{aligned}
$$

where the left-hand term of the inequality

$$
\sum_{\mathrm{j}=1}^{\mathrm{n}} a_{i j} x_{j} \geq y_{i}
$$

represents the number of zones in which cancer is detected for prostate $\mathrm{i}$. If this number is zero, then the inequality forces $y_{i}$ to be zero. Otherwise, if this number is one or larger, the inequality permits $y_{i}$ to be either 0 or 1 . Since the objective here is to maximize the sum of $y_{i}$ variables, the optimal solutions will have $y_{i}=1$.

Problems (P1) and (P2) can be modified to include various physician preferences. For example, to impose a left-right symmetry in the protocols, we can add the constraints $x_{l}=x_{r}$ for every pair of zones $l$ and $r$ that are symmetrical in the distribution map. To restrict biopsies to zones in the posterior area (between urethra and rectum in prostate anatomy), we can impose the condition $x_{a}=0$ for all zones in the anterior area. We have investigated a number of strategies for the optimal biopsy protocols, and some examples are shown below. We have used the software package ILOG CPlex $6.5^{9}$ to solve the relevant integer programs. In all cases, the CPlex solved the problems within seconds on a variety of platforms.

\section{(1) Protocols with $100 \%$ Detection Rate}

Without constraint:

- rlp2-base, lma1-base, lmp1-mid, rlp2-mid, lma2-mid, lmp2-apex, rlp2-apex, rma1-apex

- llp1-base, llp2-base, rma1-base, lmp2-mid, rlp2-mid, lmp1-apex, rlp2-apex, lma1-apex

With constraint (limit to symmetric zones in posterior 1 and 2, and anterior 1):

- llp2/rlp2-base lmp1/rmp1-mid, lla1/rla1-mid, llp2/rlp2-apex, lla1/rla1-apex

- lla1/rla1-base lmp1/rmp1-mid, lla1/rla1-mid, llp2/rlp2-apex, lma1/rma1-apex

\section{(b) Protocols with Given Number of Biopsies}

With constraint (limit to 8 symmetric zones in posterior 1 and 2, and anterior 1):

- llp2/rlp2-base lmp1/rmp1-mid, lla1/rla1-mid, llp2/rlp2-apex (Rate: 280/281)

- llp2/rlp2-base lma1/rma1-mid, lla1/rla1-mid, llp2/rlp2-apex (Rate: 280/281)

With constraint (limit to 6 symmetric zones in posterior 1 and 2, and anterior 1):

- lma1/rma1-base llp2/rlp2-mid, lmp2/rmp2-apex (Rate: 272/281) 
With constraint (limit to 10 symmetric zones in posterior 1 and 2):

- llp2/rlp2-base, lmp1/rmp1-mid, llp2/rlp2-mid, lmp2/rmp2-mid, llp2/rlp2-apex (Rate: 275/281)

With constraint (limit to 8 symmetric zones in posterior 1 and 2):

- llp2/rlp2-base, lmp1-rmp1-mid, llp2/rlp2-mid, lmp2/rmp2-apex (Rate: 274/281)

With constraint (limit to 6 symmetric zones in posterior 1 and 2):

- llp2/rlp2-base, llp2/rlp2-mid, lmp2/rmp2-apex (Rate: 271/281)

Some of these results are supporting new protocols recently proposed by urologists. Note that here when there are multiple protocols for the same constraint, it means that they have equal capability of detecting cancer in theory, and it is up to the urologists to decide which one is more appropriate to use with respect to the specific patients. Ideally, an optimized biopsy protocol will achieve maximized detection rate of clinically significant cancer with minimized number of needles at accessible entry locations. At present, we are concentrating only on detecting prostate cancer regardless of its clinical significance. The significance of detected prostate cancer can be later determined in the staging step. In the future, however, both diagnosis and staging of prostate cancer will be addressed by developing unified optimal biopsy protocols. The developed optimal biopsy protocols will be compared and evaluated against those that are currently widely used clinically. The evaluation process will be performed first by computer simulation using 3D prostate models that were not involved during optimization, and then by a limited clinical trial on patients who are recommended for prostate needle core biopsies.

\section{Conclusions}

Performance of prostate needle biopsy can be significantly improved by providing the urologists with more information on cancer distribution. This research has revealed that such distribution can be precisely calculated using modeling and mapping techniques. We have successfully modeled prostate cancer using real prostate specimens with localized cancer. We have also developed a 3D distribution map of prostate cancer based on which optimized biopsy protocols have been developed. This is the first time ever that prostate needle biopsy protocols have been quantitatively developed and optimized based on a large database of computerized real prostate specimens. Once finally evaluated, the developed biopsy protocols could have great impact on clinical practice for improved outcome of diagnosis of prostate cancer.

Currently, we are also using the developed cancer distribution map and the optimized biopsy protocols to develop an image-guided in vivo online system for real patient biopsy. Techniques developed in this research are expected to be applicable to many other clinical procedures such as radiation therapy of prostate cancer. 


\section{References}

1. Bankhead C. Sextant biopsy helps in prognosis of Pca, but it's not foolproof. Urology Times 1997; 25(8)

2. Bauer JJ, Zeng J et al. 3D Computer simulated prostate models: Lateral prostate biopsies increase the detection rate of prostate cancer. Urology 1999; 53(5):961-967

3. Chang JJ, Shinohara K et al. Prospective evaluation of lateral biopsies of the prostate for cancer detection. J. Urol. 1998; 159(5):179, abstract \# 688

4. Daneshgari F, Taylor GD et al. Computer simulation of the probability of detecting low volume carcinoma of the prostate with six random systematic core biopsies. Urology 1995; 45: 604-609

5. Egevad L, Frimmel H et al. Three-dimensional computer reconstruction of prostate cancer from radical prostatectomy specimens: Evaluation of the model by core biopsy simulation. Urology 1999; 53: 192-198

6. Eskew AL, Bare RL, McCullough DL. Systematic 5-region prostate biopsy is superior to sextant method for diagnosing carcinoma of the prostate. J. Urol. 1997; 157: 199-202

7. Goto $\mathrm{Y}$, Ohori $\mathrm{M}$ et al. Distinguishing clinically important from unimportant prostate cancers before treatment: value of systematic biopsies. J. Urol. 1996; 156: 1059-1063

8. Hodge KK, McNeal et al. Random systematic versus directed ultrasound guided transrectal core biopsies of the prostate. J. Urol. 1989; 142: 71-74

9. ILOG CPLex 6.5 User Manual, ILOG, 1999

10. Kaplan CR, Zeng J et al. Comparison of sextant to 5-region biopsy technique using a 3D computer simulation of actual prostate specimens. J. Urol. 1998; 159(5):179, abstract \# 687

11. Lin W, Liang C, Chen C. Dynamic elastic interpolation for 3D medical image reconstruction from serial cross section, IEEE Trans. Medical Imaging 1988; 7(3): 225 232

12. Nash SG, Sofer A. Linear and Nonlinear Programming. McGraw Hill, 1996

13. Nemhauser G, Wolsey L. Integer, Combinatorial Optimization. Wiley, 1988

14. Xuan J, Sesterhenn I et al. Surface reconstruction and visualization of the surgical prostate model. SPIE Medical Imaging 1997, Vol. 3031. San Diego 50-61

15. Zeng J, Kaplan CR et al. Optimizing prostate needle biopsy through 3D simulation. SPIE Medical Imaging 1998, Vol. 3335. San Diego 488-497 